\title{
Flexible PZT thin film transferred on polymer substrate
}

Thibault Dufay ${ }^{\text {a }}$, Benoit Guiffard ${ }^{\text {a }}$, Raynald Seveno ${ }^{\text {a }}$, Stephane Ginestar ${ }^{\text {a }}$ and Jean-Christophe Thomas ${ }^{\mathrm{b}}$

${ }^{1}$ Université Bretagne Loire, Université de Nantes, IETR UMR CNRS 6164, Faculté des Sciences et des Techniques, BP 92208, 2 rue de la Houssinière, 44322 Nantes Cedex 3, France

${ }^{2}$ Université Bretagne Loire, Université de Nantes, GeM UMR CNRS 6183, Faculté des Sciences et des Techniques, BP 92208, 2 rue de la Houssinière, 44322 Nantes Cedex 3, France

Corresponding Author: Thibault Dufay, thibault.dufay@univ-nantes.fr

\begin{abstract}
Highly flexible lead zirconate titanate, $\mathrm{Pb}(\mathrm{Zr}, \mathrm{Ti}) \mathrm{O}_{3}(\mathrm{PZT})$, thin films have been realized by an all chemical process on a plastic substrate. The procedure is composed of three steps: the first one is the PZT deposition on aluminium thin substrate, the second step corresponds to the bonding PZT thin film to a polymer layer and the final step is the aluminium substrate etching. Diffractions and microscopy techniques were used to check the quality of the new PZT/polymer composite structure. Polarization hysteresis and permittivity measurements were also performed to determine the electrical characteristics of the composite. These results demonstrate that the recently developed PZT/polymer thin films are very attractive for both bending actuation and sensing as well as for low frequency vibrating energy harvesting applications.
\end{abstract}

Keywords : Flexible PZT, Polymer substrate, Transfer process, Ferroelectric properties 


\section{Introduction}

Nowadays, flexible technologies are in great expansion and become new development trend in many evolving electronic devices such as displays, sensors, solar cells and mechanical energy harvesters. In the particular field of low frequency $(<100 \mathrm{~Hz})$ vibration energy harvesting with piezoelectrics, recent works have focused on the realization of thin PZT layer on flexible and insulating substrates [1]-[3]. Thus, the challenge is to obtain a piezoelectric ceramic material requiring high temperatures $\left(>600^{\circ} \mathrm{C}\right)$ to crystallize on a polymer substrate unable to withstand such temperatures. To address such a technological issue, the two possible options are to develop a complete low temperature process or to transfer piezoelectric material from growth substrate to polymer substrate.

Recently, our group has attempted to design reliable techniques for the fabrication of thinlayer piezoelectric structures on flexible support which resists to higher temperatures. Thin films of lead zirconate titanate (PZT), one of the most investigated and high performance piezoelectric materials, have been prepared by using chemical deposition on a flexible metallic substrate, a commercial aluminium (Al) foil with thickness less than $30 \mu \mathrm{m}$ [4]. This fabrication process is cost effective and the lightweight of the micro-generator makes it sensitive to air flow. This structure has been thoroughly characterized [5], [6] and shown promising results for energy harvesting under mechanical stress [7].

To go further, we want to achieve a structure without ground plane to be free to choose the electrode design. That is the reason why PZT films must be separated from the aluminium substrate.-However, PZT thin films are not enough rigid to stand alone, so a new substrate has to be used with a good flexibility and insulating properties. Specific polymer materials like some thermoplastics are good candidates for the new insulating and flexible substrate whose flexural rigidity can be tuned according to the selected family of polymers and the dimensions of the layer. The major drawback of these soft polymers is their low resistance against thermal 
treatment that prevents from the direct PZT deposition onto the polymeric substrate. The proposed solution is to transfer the PZT thin film, obtained by classical methods on rigid substrates, onto the polymeric substrate. This solution was used to realize PZT on polymer, but the method employed - laser lift-off - is expensive and could be difficult to transfer to the industry. [1]-[3]

In this study, we will focus on the development of a new procedure to achieve piezoelectric PZT thin film on a flexible polymer substrate. Starting from the PZT/Al thin film developed in the laboratory, a chemical process is used to transfer the piezoelectric material to a polymer substrate. This process is cheap and simple and would be easily transferred to the industry. In addition, with this chemical method, the metallic substrate is removed from the complete surface $\left(\sim 6 \mathrm{~cm}^{2}\right)$ of PZT thin film in one step whereas the laser lift-off method requires several steps to separate PZT from the first substrate.

Thus, this paper mainly deals with the method of realization of flexible PZT thin film without ground plane and comprises the chemical details to transfer a PZT thin film from aluminium substrate to a polymer substrate. The morphological, structural, compositional (surface electron microscopy (SEM), X-ray diffraction (XRD), atomic force microscopy (AFM)) characterizations of the PZT/polymer thin film are also presented to confirm the good quality of the transferred PZT thin film. Finally, comparisons of dielectric and ferroelectric characteristics of MIM structures before and after the transfer on polymer substrate are realized to assess the influence of the transfer process on the active layer properties.

\section{Material and methods}

PZT thin layer fabrication begins with the preparation of a precursor solution which is obtained by mixing different chemical products. Initially, lead acetate is dissolved in a solution of acetic acid and then zirconium n-propoxide and titanium n-propoxide are added in 
desired proportions. The final precursor solution will be ready by adding ethylene glycol, which limits the crack formations in PZT thin film during its thermal treatment.

The precursor solution is then deposited by spin-coating technique on a sacrificial substrate, an aluminium thin foil, which can be etched in the following by simple chemical process. A stainless steel support is used in order to facilitate the spin-coating deposition. This technique allows the formation of a piezoelectric film on the complete surface of the aluminium substrate. The rotation speed and spinning duration are chosen according to the desired thickness of thin layer. After each spin-coating step, the material is subjected to a thermal treatment at temperature of $650{ }^{\circ} \mathrm{C}$ for the duration of 2 minutes to crystallize the PZT thin layer. In practice, the thickness of the PZT thin layer obtained after the execution of one spincoating step is $300 \mathrm{~nm}$. Thus, to form a thicker PZT thin film, deposition step can be reiterated as many times as necessary to obtain a thin film of several micrometers.

The different steps of the PZT transfer process are schematically represented in Figure 1.

Platinum (Pt) top electrode (200 nm thick) is added on the whole surface of PZT. It will be used as the back electrode after the transfer on polymer substrate. Platinum has been chosen for its good resistance against chemical etching.

A thin adhesive layer of polyurethane (PU, NOA81, Norland Optics $\left.{ }^{\circledR}\right)$ is deposited on the PZT thin film by spin-coating. Once the PU adhesive layer is added, it is open with acetone to ensure electric contact with Pt after transfer. The adhesive layer is then cured under UV exposure during 2 hours. It ensures a good binding between the PZT and polymeric materials. Polyurethane NOA81 has elastic nature which is required for the fabrication the flexible piezoelectric structure. 


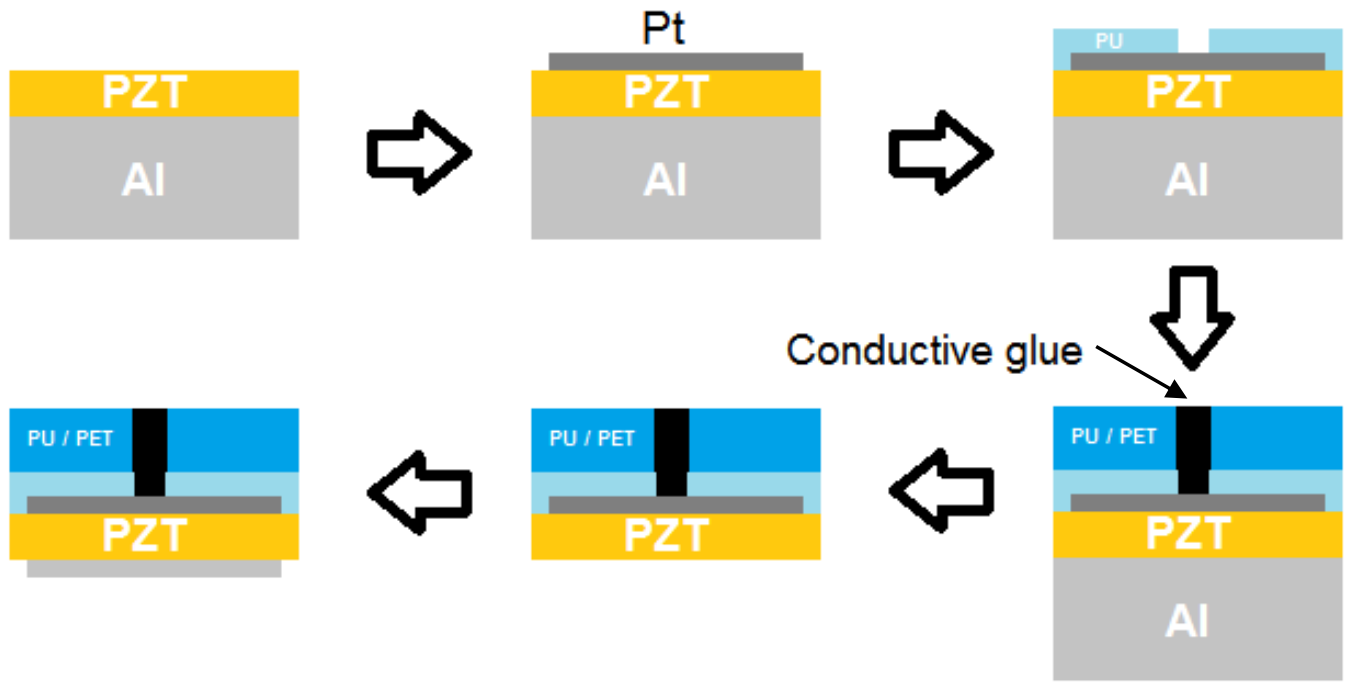

Figure 1 : Flow chart of the process of PZT transfer.

Thick elastic polymer layer is attached on the PU adhesive to form the permanent substrate of the final flexible piezoelectric structure. This step is carried out using a thermofusing technique. The sample is heated while a small pressure is applied, to create a good binding between the two polymers layers. Commercial polyethylene terephthalate (PET) is a suitable thermoplastic material for new substrate because it is an elastically deformable and low-cost polymer. Furthermore, several thicknesses could be used to get the desired mechanical properties.

The new substrate has to be opened before the binding in order to realize a kind of electric via by the filling with conductive glue. This step is also very important for the realisation of MIM structure onto a polymer substrate because it reinforces the whole structure. Indeed, the connection channel created through the polymer substrate gives fragility at this specific point to the PZT layer. Macroscopic cracks could happen during the etching step. So, the conductive glue is needed to bring a better mechanical support to PZT. The glue has to resist to the chemical etching solution to preserve the electric contact on back $(\mathrm{Pt})$ electrode.

At this point, the obtained structure is comprised of aluminium sacrificial substrate, PZT active layer, conductive glue, PU adhesive layer and polymeric substrate. This structure is 
immersed in an iron chloride solution of formula $\mathrm{FeCl}_{3}$ to undergo a selective chemical etching of the aluminium sacrificial substrate. The chemical agent $\mathrm{FeCl}_{3}$ is chemically inactive in presence of piezoelectric and polymeric materials (PZT, PU and PET). This step is done in less than 5 minutes with a pure iron chloride solution at room temperature. The final step consists in depositing an aluminium electrode ( $200 \mathrm{~nm}$ thick) to achieve a MIM structure.

A basic flexible piezoelectric structure is obtained after sacrificial substrate is dissolved. It is composed of a piezoelectric thin film arranged on a stack of elastically deformable polymeric layers which is used as the flexible permanent substrate. This structure is free of stiff supporting layer acting as ground plane and completely flexible. Both of the two discussed structures (PZT on $\mathrm{Al}$ and on PET) are presented in Figure 2.

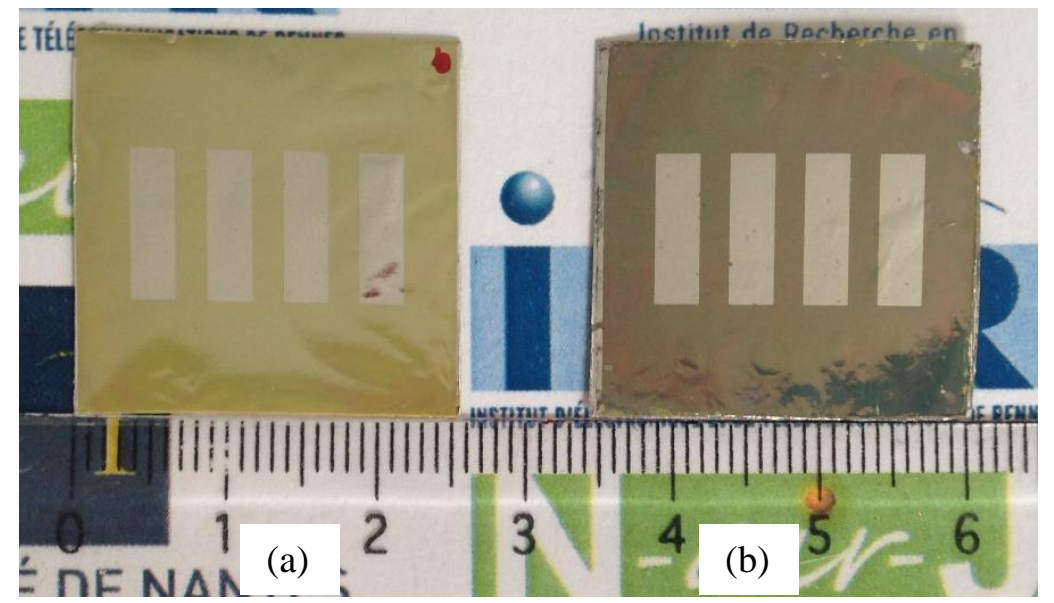

Figure 2: Photos of (a) PZT/Al and (b) PZT/PET samples.

Atomic Force Microscopy (AFM) was used to measure the roughness of the aluminium substrate (before and after a heat-treatment at $650{ }^{\circ} \mathrm{C}$ for $2 \mathrm{~min}$ ) and the PZT surface (area of $95 \mu \mathrm{m} \times 95 \mu \mathrm{m})$. Polarisation-Electric field (P-E) measurements have been done using a Sawyer-Tower circuit with a $4.81 \mu \mathrm{F}$ serial capacitor, and the applied sinusoidal electric field amplitude was maintained around $300 \mathrm{kV} / \mathrm{cm}$ in order to see the difference in remanent polarization $P_{r}$ and electric coercive field $E_{C}$ for PZT films deposited onto aluminium and 
transferred onto PET. The permittivity and the dielectric losses have been measured by using a HP 4275A LCR meter between 100 and $10^{5} \mathrm{~Hz}$.

\section{Results and discussion}

The above described method proves that the transfer of the piezoelectric thin layer onto a flexible substrate is possible just by using a simple chemical method. The obtained piezoelectric thin film on polymer substrate has been characterized with XRD, SEM and AFM as complementary techniques. MIM structure was realized to investigate ferroelectric properties of the transferred PZT thin film.

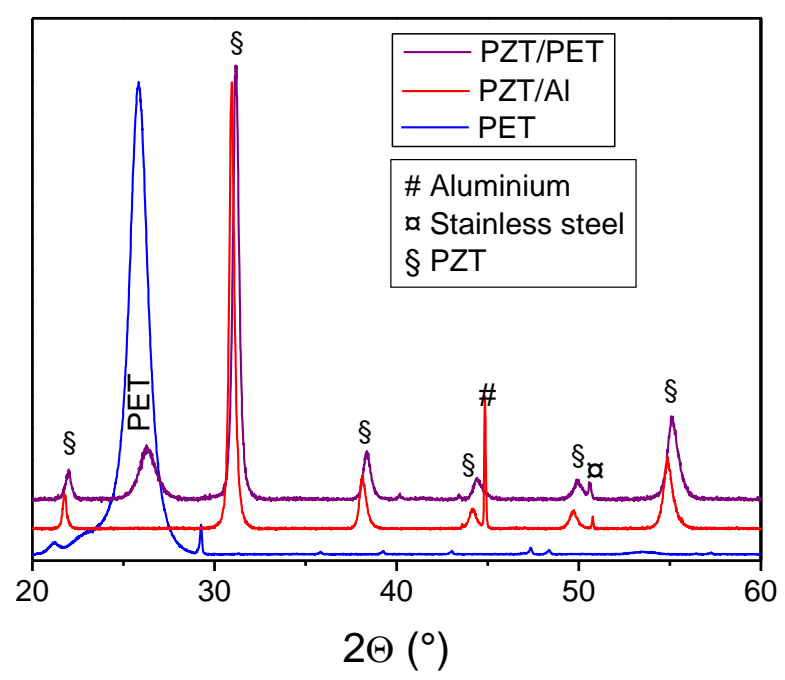

Figure 3 : XRD patterns of PZT/Al, PZT/PET and PET.

XRD patterns of PZT/Al and PZT/PET are roughly the same except for the substrates peak.

Indeed the peak of aluminium is replaced by the peak of PET, but all the peaks of PZT remain at their positions. The position of the PET peak was verified by an investigation on a single PET layer. The slight difference in $2 \Theta$ angle observed is a consequence of the flexible substrates, the base surface being not plane in the both cases leading to shifts in $2 \Theta$ angle.

AFM tip deflection was shown for the aluminium substrate and transferred PZT thin films in a previous paper [8]. The roughness was measured on the whole surface for the two faces of the 
aluminium. A large surface was chosen in order to be sure to deposit a sufficiently thick metal layer in order to ensure electrodes continuity on PZT. The results of roughness measurements are presented in Table 1. It is found that the roughness of aluminium face is transferred on the PZT layer revealed by the transfer. The flexibility of aluminium and PET could induce uncertainty in the roughness measurements which may also explains the differences observed between the aluminium faces and the first PZT layer.

Table 1 : Roughness of aluminium substrate faces and PZT layer revealed by the transfer process.

\begin{tabular}{c|cc}
\hline Roughness (nm) & Brillant face & Mat face \\
\hline Aluminium & 150 & 310 \\
PZT & 180 & 320 \\
\hline
\end{tabular}

Cross-section SEM image of transferred PZT presented in Figure 4 was realized to show the good binding between PZT and the two layers of polymer. The adhesion of the ceramic layer on the adhesive polymer is very good and a strength bonding is observed between the two polymers.

The transferred MIM structure (PZT/PET) is compared to the Al/PZT/Al structure in order to show the influence of the transfer process on active material properties. Ferroelectric and dielectric characterizations were realized on both PZT/Al and PZT/PET MIM structures. 


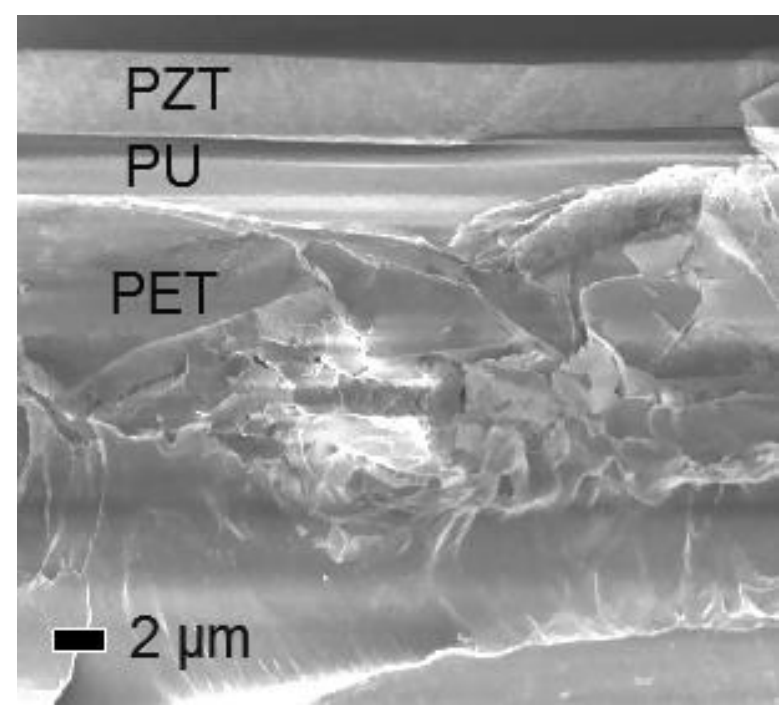

Figure 4 : Cross-section SEM image of PZT transferred on polymer substrate.

P-E loops of PZT/Al and PZT/PET are showed in Figure 5. A lower $P_{r}$ value is observed for PZT/PET with $16 \mu \mathrm{C} / \mathrm{cm}^{2}$ against $21 \mu \mathrm{C} / \mathrm{cm}^{2}$ for PZT/Al. This is combined with a slightly higher $E_{C}$ of $130 \mathrm{kV} / \mathrm{cm}$ for PZT/PET compared to $115 \mathrm{kV} / \mathrm{cm}$ for PZT/Al. Depending on ferroelectric properties, it seems that the transfer process has an undesirable impact on the PZT with minor degradation of $P_{r}$ and $E_{C}$.

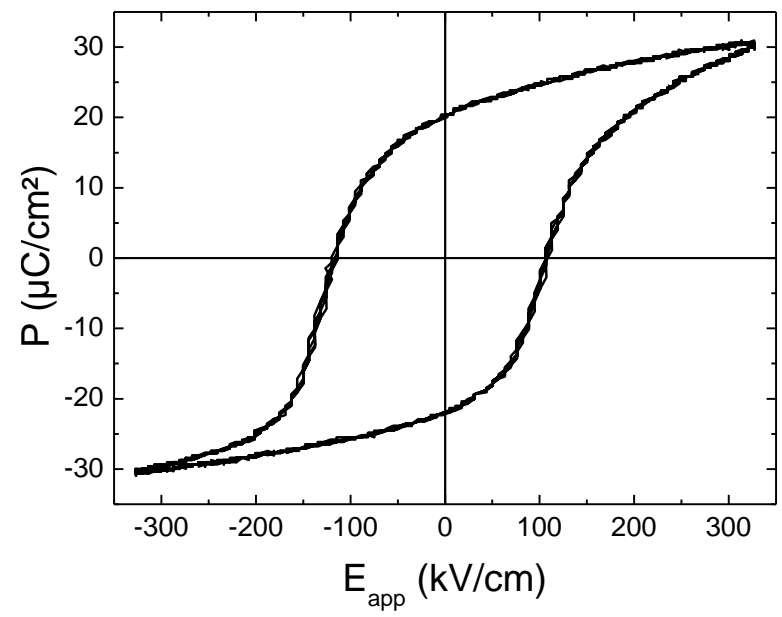

(a)

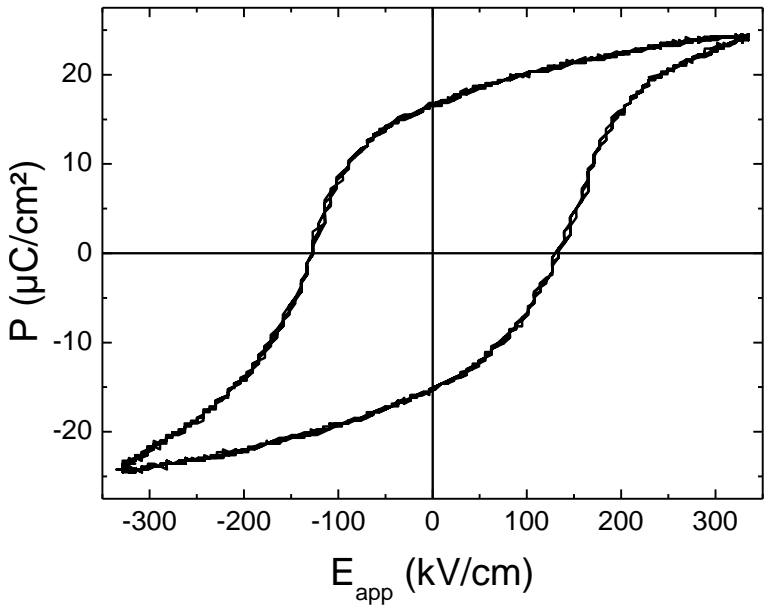

(b)

Figure 5 : Hysteresis loops of (a) Al/PZT/Al and (b) Al/PZT/Pt/NOA81/PET films.

Dielectric measurements of the dielectric relative permittivity and dielectric losses are presented in Figure 6. Dielectric loss factor $\tan \delta$ are quite similar for the two samples and remain stables at low values of 0.02 and 0.03 . A little difference is observed in $\varepsilon_{r}$ with a 
decrease of this parameter after the transfer (335 and 270). The minor degradations due to the transfer process also modify dielectric properties. Whereas tan $\delta$ is not much impacted with the transfer, the relative dielectric permittivity is modified by the transfer.

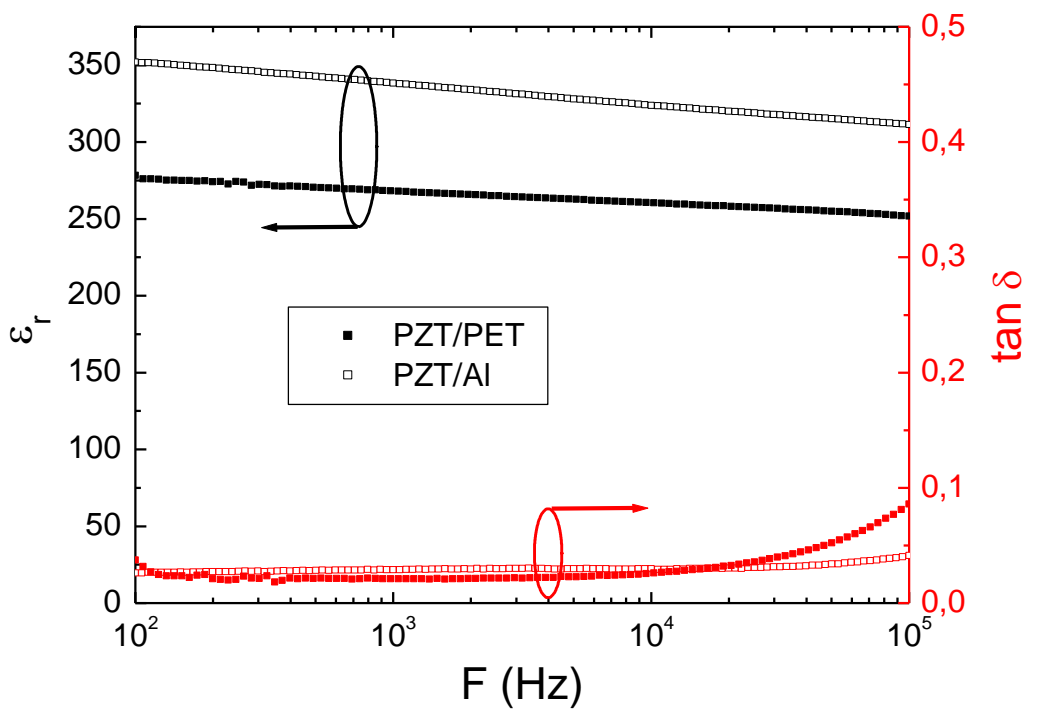

Figure 6 : Measurements of dielectric relative permittivity and losses for PZT on aluminium substrate and transferred onto PET.

This could be explained by a small degradation of the PZT during the etching step. During the development of the process, aluminium electrode was tested instead of platinum for back electrode after transfer. During etching step, iron chloride solution goes through the active layer because of its porosity and damages the aluminium electrodes. With this information, it may be reasonably assumed that the porosity could rise due to interaction with etching solution.

This explanation is strengthened by the fact that many samples were in short-circuit, indicating a lower insulating quality of the PZT layer. In addition, PZT properties could decrease with a wet etching process [9], [10]. In those reported cases, the PZT is structured by chemical etching and implies a degradation of the ferroelectric and piezoelectric properties.

The laser lift-off (LLO) transfer method used by Do et al.[1] produces a very slight decrease in ferroelectric properties. For example, remnant polarisation $\operatorname{Pr}$ is $27 \mu \mathrm{C} / \mathrm{cm}^{2}$ before transfer 
and $23 \mu \mathrm{C} / \mathrm{cm}^{2}$ after. In the same time, the relative dielectric permittivity is measured at 1089 and 1046, respectively before and after LLO transfer. Apparently, this quite sophisticated method gets better results and lower decrease of the properties. But the LLO transfer requires more specific equipments and time. What we proposed is a simple alternative method to this highly technical process. Quite good results are obtained with a shorter experimental time.

Besides, the new structure is functional and could be of great interest for some flexible applications like sensors or actuators. In addition, this is the first step to the realization of interdigitated electrodes in order to develop efficient low frequency vibration-based energy harvesters.

\section{Conclusion}

A new process for PZT transfer to polymer substrate is described in this paper. The use of chemical solution deposition for the realization of the PZT on sacrificial substrate is complementary with chemical technics developed for the transfer process. The entire process is then easy to industrialize. Structural characterizations were realized to ensure the good binding between the different layers after the transfer. The crystallinity of the PZT was also checked before ferroelectric and dielectric measurements. Before and after transfer process, comparison was done for those electrical measurements revealing a small degradation of properties due to the etching step. The explanation proposed is that the PZT porosity is enhanced because of the interaction with iron chloride solution. Nevertheless, characteristics of PZT/PET composite are very interesting and this structure will be investigated for sensing and energy harvesting applications.

\section{Acknowledgments}

The authors would like to thank Jean-Emmanuel Lechêne from Cookson SAS (Cholet, France) for his receptiveness to supply the shadow masks required for electrode deposition. 
This work is supported by the French region Pays de la Loire through the 201407965 contract. 
[1] Y. H. Do, M. G. Kang, J. S. Kim, C. Y. Kang, and S. J. Yoon, 'Fabrication of flexible device based on PAN-PZT thin films by laser lift-off process', Sens. Actuators Phys., vol. 184, pp. 124-127, Sep. 2012.

[2] Y. H. Do, W. S. Jung, M. G. Kang, C. Y. Kang, and S. J. Yoon, 'Preparation on transparent flexible piezoelectric energy harvester based on PZT films by laser lift-off process', Sens. Actuators Phys., vol. 200, pp. 51-55, Oct. 2013.

[3] K.-I. Park et al., 'Highly-Efficient, Flexible Piezoelectric PZT Thin Film Nanogenerator on Plastic Substrates', Adv. Mater., vol. 26, no. 16, pp. 2514-2520, Apr. 2014.

[4] R. Seveno and D. Averty, 'Ultra light tunable capacitor based on PZT thin film deposited onto aluminium foil', J. Sol-Gel Sci. Technol., vol. 68, no. 2, pp. 175-179, Nov. 2013.

[5] T. Dufay, B. Guiffard, J.-C. Thomas, and R. Seveno, 'Transverse piezoelectric coefficient measurement of flexible lead zirconate titanate thin films', J. Appl. Phys., vol. 117, no. 20, p. 204101, May 2015.

[6] R. Seveno, J. Carbajo, T. Dufay, B. Guiffard, and J. C. Thomas, 'Flexible PET/Al/PZT/Al/PET multi-layered composite for low frequency energy harvesting', $J$. Phys. Appl. Phys., vol. 50, no. 16, p. 165502, 2017.

[7] R. Seveno, B. Guiffard, T. Dufay, and J. C. Thomas, 'Flexible PZT/aluminium thin films characterizations for energy harvesting at very low frequencies $(\sim 1 \mathrm{~Hz})$ ', in Applications of Ferroelectric, International Symposium on Integrated Functionalities and Piezoelectric Force Microscopy Workshop (ISAF/ISIF/PFM), 2015 Joint IEEE International Symposium on the, 2015, pp. 94-97.

[8] T. Dufay, R. Seveno, B. Guiffard, and J. C. Thomas, 'New process for transferring PZT thin film onto polymer substrate', in 2016 Joint IEEE International Symposium on the Applications of Ferroelectrics, European Conference on Application of Polar Dielectrics, and Piezoelectric Force Microscopy Workshop (ISAF/ECAPD/PFM), 2016, pp. 1-4.

[9] T.-H. Yeh, M. B. Suresh, J.-N. Shen, J.-C. Yu, and C.-C. Chou, 'Chemical Reactions During Wet-Etching Process of LSMO/PZT/LSMO-Structured Device Fabrication', Ferroelectrics, vol. 380, no. 1, pp. 97-101, Jun. 2009.

[10] T. Kobayashi, M. Ichiki, R. Kondou, K. Nakamura, and R. Maeda, 'Degradation in the ferroelectric and piezoelectric properties of $\mathrm{Pb}(\mathrm{Zr}, \mathrm{Ti}) \mathrm{O} 3$ thin films derived from a MEMS microfabrication process', J. Micromechanics Microengineering, vol. 17, no. 7, p. 1238, 2007. 


\section{Figure captions}

Figure 1 : Flowchart of the process of PZT transfer.

Figure 2 : Photos of (a) PZT/Al and (b) PZT/PET samples.

Figure 3 : XRD patterns of PZT/Al, PZT/PET and PET.

Figure 4 : Cross-section SEM image of PZT transferred on polymer substrate.

Figure 5 : Hysteresis loops of (a) Al/PZT/Al and (b) Al/PZT/Pt/NOA81/PET films.

Figure 6 : Measurements of dielectric relative permittivity and losses for PZT on aluminium substrate and transferred onto PET. 
Figures

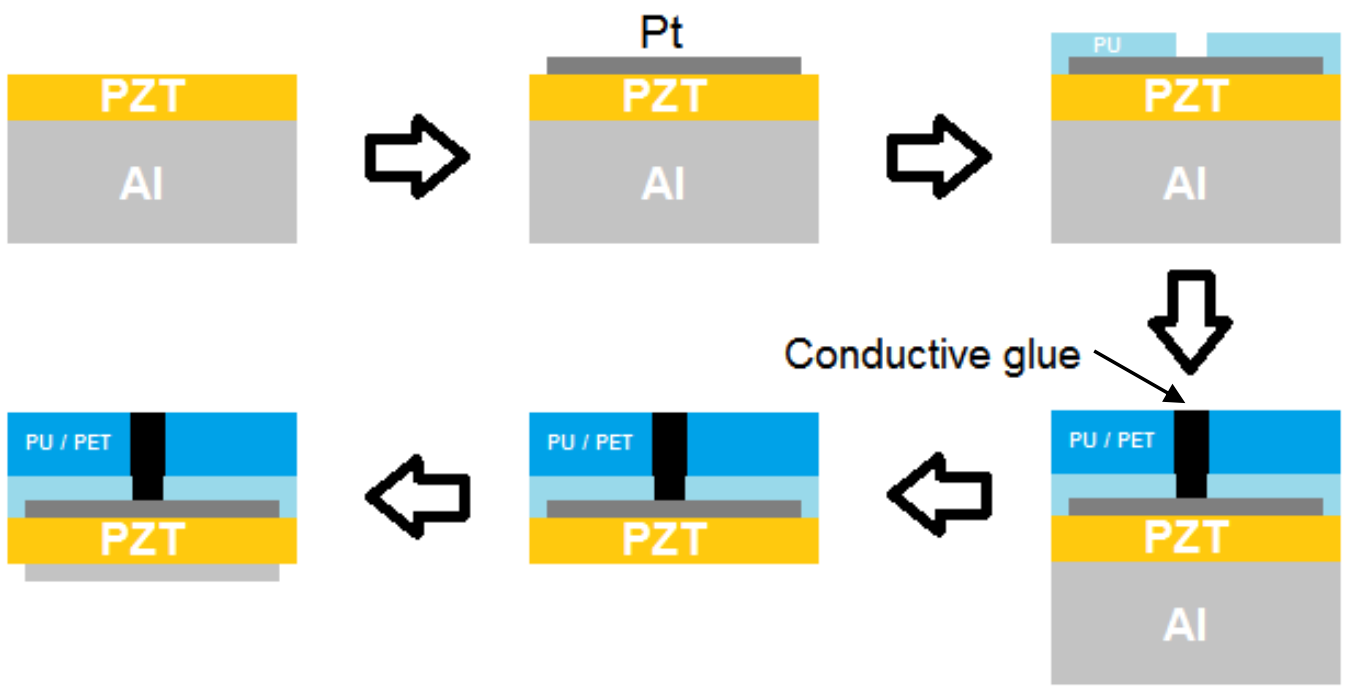

1

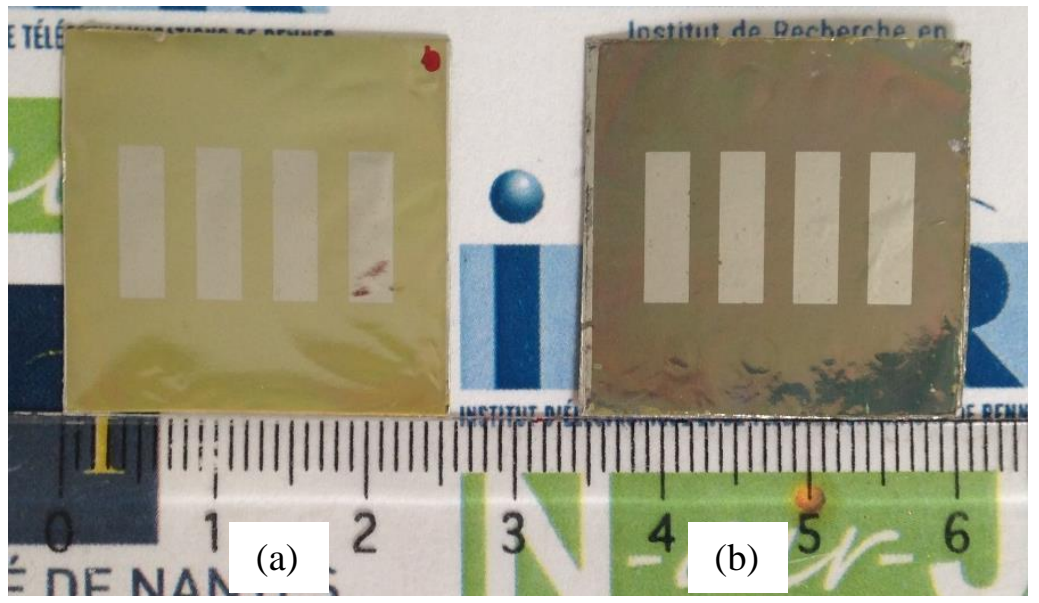

2 

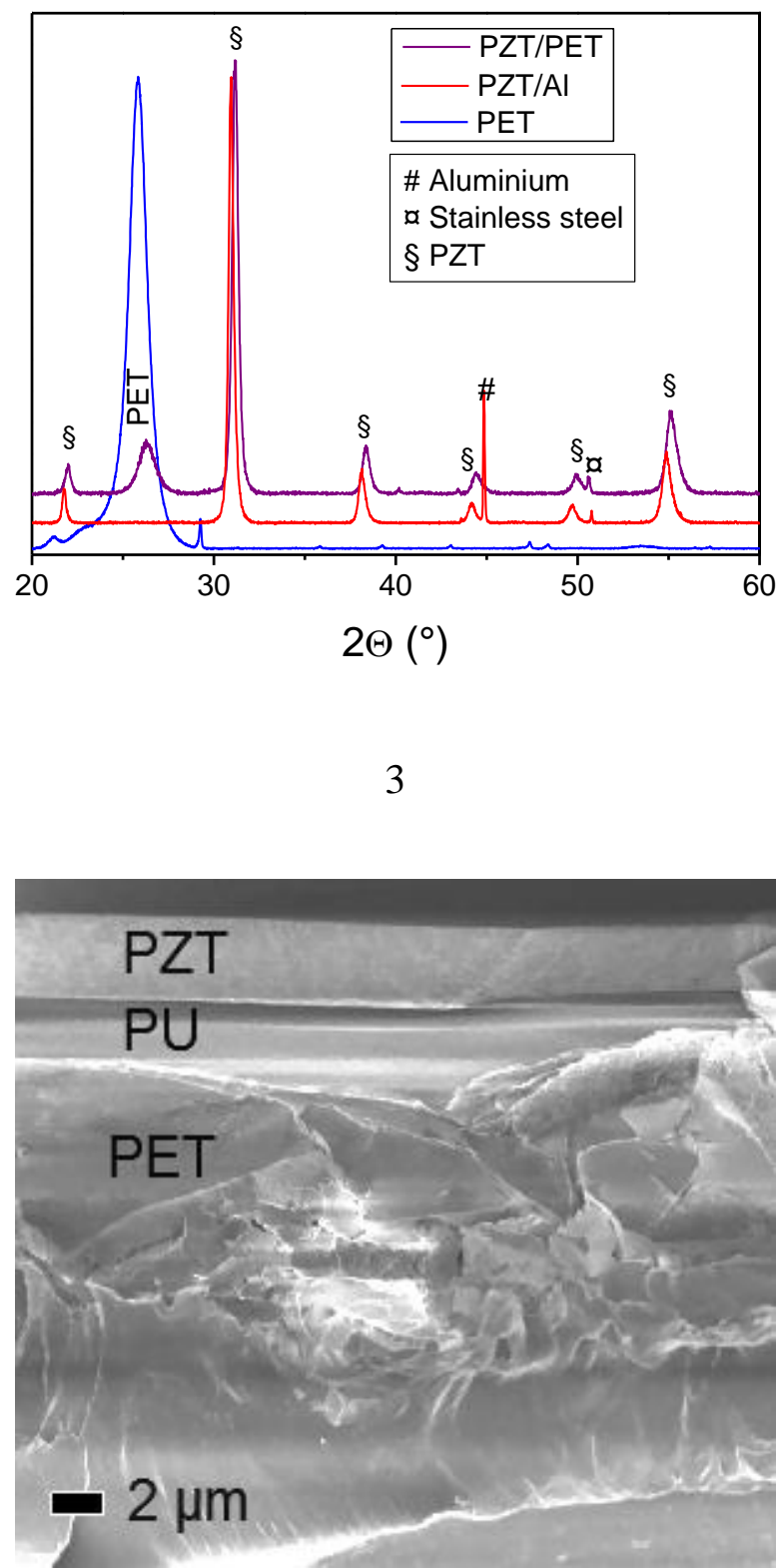


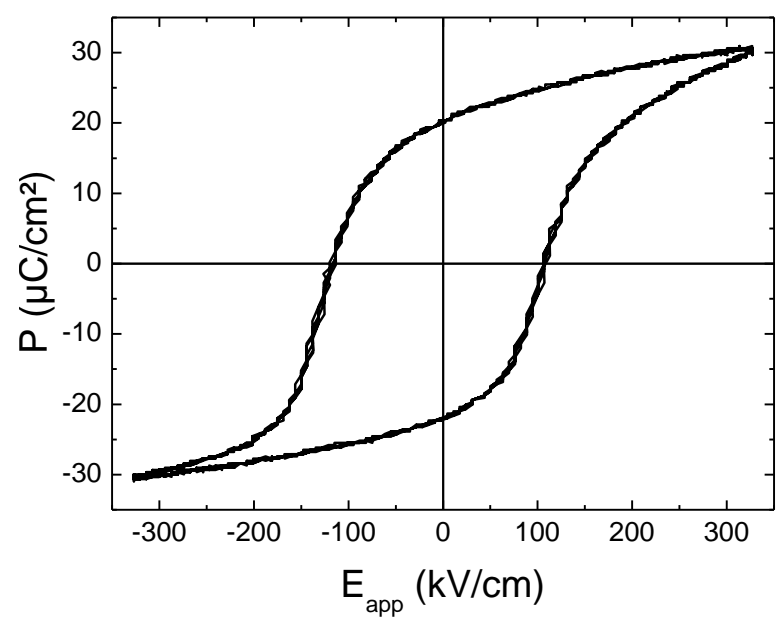

(a)

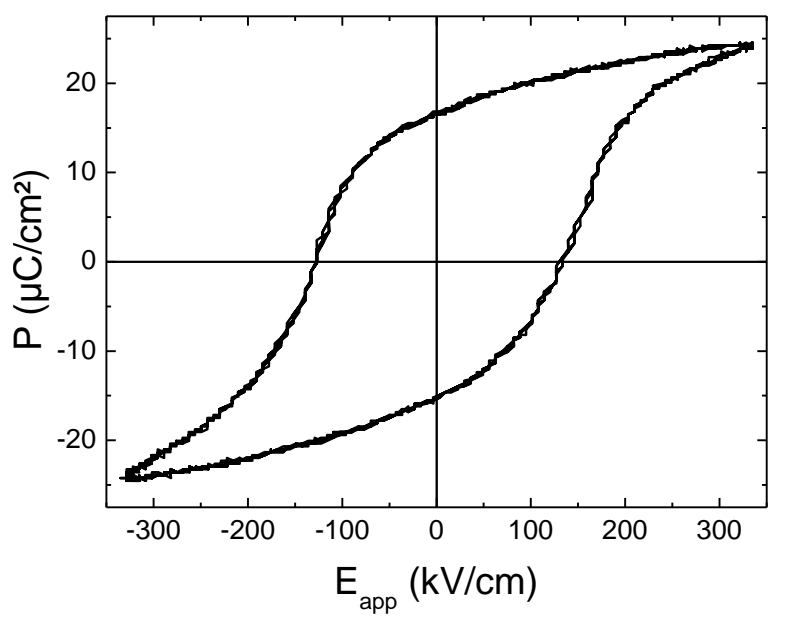

(b)

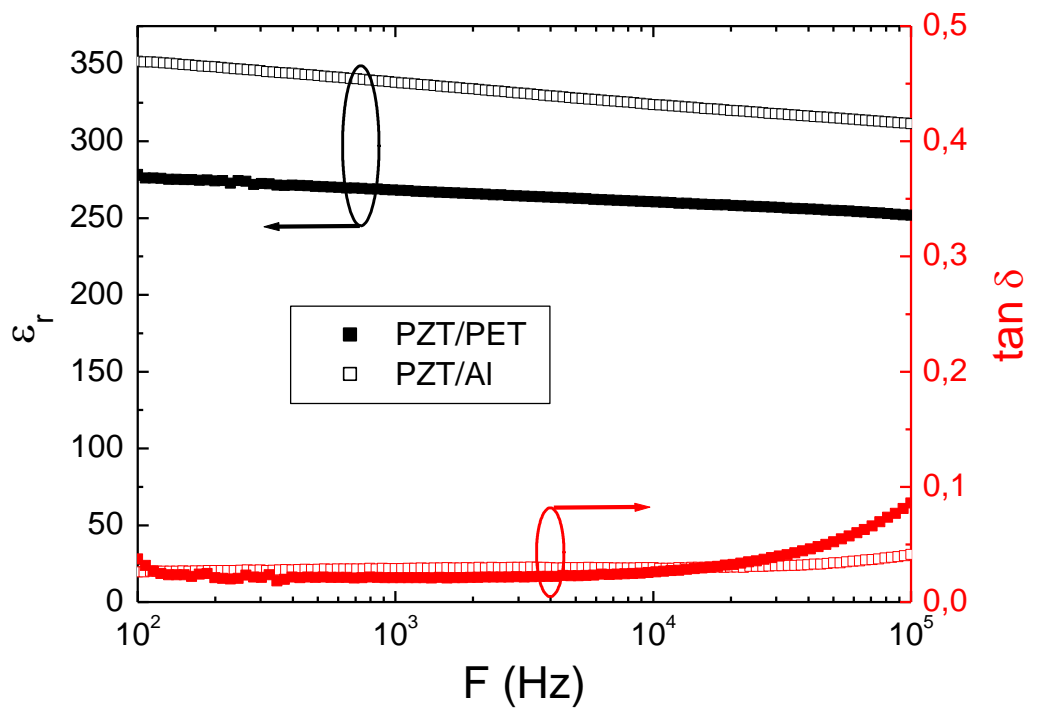

6 
Highlights:

- PZT transfer process on polymer substrate based on chemicals methods.

- The good binding of PZT on polymer substrate is ensure with SEM images.

- Comparison of electric properties before and after the transfer are realized.

- A small degradation is observed due to the interaction between PZT and the etchant. 